\title{
Functionalized Synthetic Biodegradable Polymer Scaffolds for Tissue Engineering
}

\author{
Xiaohua Liu, Jeremy M. Holzwarth, Peter X. Ma*
}

Scaffolds (artificial ECMs) play a pivotal role in the process of regenerating tissues in 3D. Biodegradable synthetic polymers are the most widely used scaffolding materials. However, synthetic polymers usually lack the biological cues found in the natural extracellular matrix. Significant efforts have been made to synthesize biodegradable polymers with functional groups that are used to couple bioactive agents. Presenting bioactive agents on scaffolding surfaces is the most efficient way to elicit desired cell/material interactions. This paper reviews recent advancements in the development of functionalized biodegradable polymer scaffolds for tissue engineering, emphasizing the syntheses of functional biodegradable polymers, and surface modification of polymeric scaffolds.

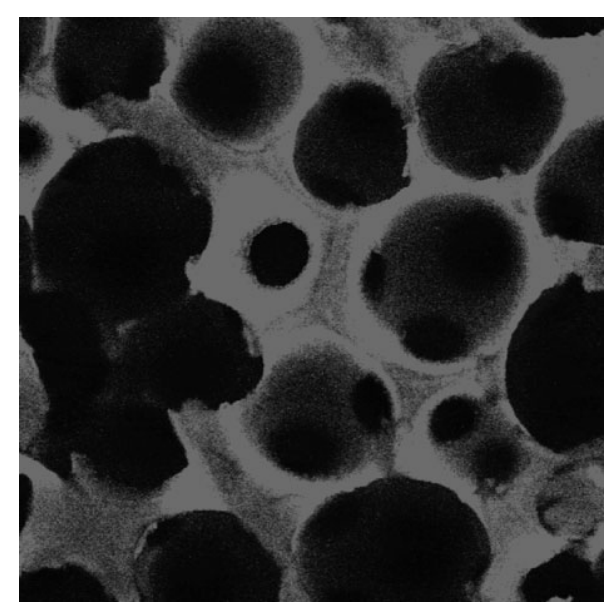

\section{Introduction}

As a multi-disciplinary field, tissue engineering integrates materials science with regenerative medicine by applying the principles of engineering and biology to clinical issues. $^{[1]}$ A typical tissue engineering strategy can be separated into three components: a scaffold [an artificial extracellular matrix (ECM)], cells, and biological factors. The

X. Liu, Dr. P. X. Ma

Department of Biologic and Materials Sciences, University of

Michigan, Ann Arbor, MI 48109, USA

E-mail: mapx@umich.edu

X. Liu

Department of Biomedical Sciences, Baylor College of Dentistry, Texas A\&M HSC, Dallas, TX 75246, USA

Dr. P. X. Ma, J. M. Holzwarth

Department of Biomedical Engineering, University of Michigan,

Ann Arbor, MI 48109, USA

Dr. P. X. Ma

Macromolecular Science and Engineering Center, and

Department of Materials Science and Engineering, University of

Michigan, Ann Arbor, MI 48109, USA scaffold serves as a template for tissue regeneration and plays a pivotal role in cell adhesion, proliferation, differentiation, and new tissue formation in three dimensions (3D). Ideally, a scaffold should be designed to possess the following characteristics: (i) a biocompatible and biodegradable substrate with controllable degradation rates; (ii) a 3D and highly porous architecture to accommodate cell attachment, penetration, proliferation, and ECM deposition; (iii) an interconnected pore network to facilitate nutrient and waste exchange; (iv) a suitable mechanical strength to support regeneration; and (v) a proper surface chemistry and surface topography to promote cellular interactions and tissue development. ${ }^{[2,3]}$ With the advancement of developmental biology and nanotechnology, recent research on scaffolding has more focused on the design and synthesis of functionalized scaffolds that can elicit desirable cell/material interactions to guide cell behavior and enhance new tissue formation. ${ }^{[4-8]}$

Scaffolds can be produced from a variety of materials, including metals, ceramics, and polymers. Metallic alloys are popular for both dental and bone implants ${ }^{[9]}$ while ceramics with good osteoconductivity have been used for 
bone tissue engineering. ${ }^{[10]}$ However, both metals and ceramics have significant drawbacks. Metals are not biodegradable and do not provide a biomimetic matrix for cell growth and tissue formation. Ceramics also have limited biodegradability and are difficult to process into highly porous structures due to their brittleness. In contrast, polymers have great processing flexibility and their biodegradability can be imparted through molecular design. Therefore, polymers are dominant scaffolding materials in tissue engineering. In general, naturally derived polymers have the potential advantage of biological recognition that may positively support cell adhesion and function. However, complexities associated with natural polymeric materials, including complex structural composition, purification, immunogenicity, and pathogen transmission, have driven the development of synthetic polymers for use as scaffolding materials. Synthetic polymers have a higher degree of processing flexibility and no immunological concerns compared to natural ECM proteins. By incorporating bioactive agents into synthetic polymers, functionalized scaffolds that combine the advantage of both synthetic and natural polymeric materials can be fabricated.

This paper covers the design and fabrication of functionalized biodegradable polymer scaffolds, focusing on the synthesis of functional biodegradable polymers and the surface modification of polymeric scaffolds. Selected examples from both our and other groups are presented for the purpose of illustration. Additionally, the cellular response on functionalized scaffolds will be briefly discussed. Since the methods of scaffolding fabrication and incorporation of growth factors into scaffolds have been extensively reviewed in detail elsewhere, ${ }^{[2,3,11-18]}$ they will not be the focus of this paper.

\section{Synthesis of Functional Biodegradable Polymers}

Poly( $\alpha$-hydroxyacids), including poly(glycolic acid) (PGA), poly(lactic acid) (PLA), and their copolymer poly[(lactic acid)-co-(glycolic acid)] (PLGA), are the most widely used synthetic polymeric materials in tissue engineering. ${ }^{[19]}$ These polymers are well characterized and have gained FDA approval for certain human use (e.g., sutures). Poly( $\alpha$ hydroxyacids) have been fabricated into 3D scaffolds via a number of techniques. For example, poly(L-lactic acid) (PLLA) has been fabricated into nano-fibrous scaffolds to mimic the physical architecture of natural collagen (a main component of ECM). ${ }^{[20]}$ The nano-fibrous PLLA scaffolds have been demonstrated to enhance cell adhesion and differentiation. ${ }^{[21,22]}$ However, there are no functional groups available on the poly ( $\alpha$-hydroxyacids) chains, which limits the capacity to incorporate biologically active

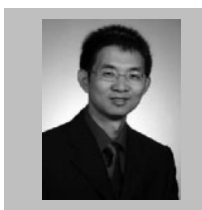

Xiaohua Liu is an Assistant Professor in the Biomedical Sciences Department, Baylor College of Dentistry, Texas A\&M Health Science Center. Prior to this, he received a PhD in Polymer Chemistry from Tsinghua University in China (2002). He then worked with Dr. Peter Ma as a postdoctoral fellow at the University of Michigan before he joined Baylor College of Dentistry. His current research interests are biomaterials design and synthesis and controlled drug delivery for biomedical applications.

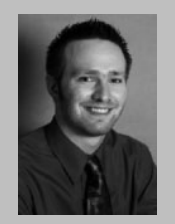

Jeremy M. Holzwarth received his BS in Chemical Engineering from Cornell University in 2009 and his MS in Biomedical Engineering from the University of Michigan in 2011. He is currently a Biomedical Engineering PhD candidate under Peter X. Ma at the University of Michigan. His research interests include the design of scaffolds for tissue engineering and the effect of nanofibrous architecture on embryonic stem cell differentiation.

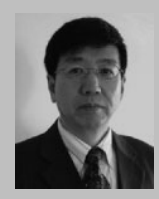

Peter X. Ma received his BS degree in Polymer Chemistry and Chemical Engineering from Tsinghua University (Beijing, China), and PhD in Polymer Science and Engineering from Rutgers University. He then did his postdoctoral research at MIT and Harvard Medical School on Biomaterials and Tissue Engineering. He is currently the Richard H. Kingery Endowed Collegiate Professor at the University of Michigan with quadruple appointments in the Department of Biologic and Materials Sciences in the School of Dentistry, the Departments of Biomedical Engineering, Materials Science and Engineering, and the Macromolecular Science and Engineering Center in the College of Engineering. He is a Fellow of the American Institute of Medical and Biological Engineering (AIMBE) and a Fellow of Biomaterials Science and Engineering (FBSE).

moieties onto the scaffolding surface. Considerable efforts have been made to improve the functionality of these polymers to further expand their applications. ${ }^{[2-5,23]}$ One strategy is to copolymerize the $\alpha$-hydroxyacids with other monomers containing functional pendant groups such as amino and carboxyl groups. In one study, L-lactide and $(R S)$ $\beta$-benzyl malate were copolymerized by ring-opening polymerization, and poly\{(L-lactide)-co-[(RS)- $\beta$-malic acid $]\}$ with pendant carboxyl groups was obtained by removing the benzyl groups. ${ }^{[24]}$ Leemhuis et al. synthesized two functionalized dilactones (benzyloxymethylmethyl glycolide and benzyloxymethyl glycolide) with protected hydroxyl groups, which were then copolymerized with $\mathrm{L}^{-}$ lactide. ${ }^{[25]}$ Deprotection of the benzyloxymethyl groups gave the corresponding hydroxylated PLLA copolymers. Noga et al. further modified the pendant hydroxyl groups of 
PLLA copolymers with succinic anhydride to obtain the corresponding carboxylic acid functionalized copolymers to attach amine-containing biological molecules. ${ }^{[26]}$ Chen et al. synthesized two cyclic carbonate monomers (acryloyl carbonate and methacryloyl carbonate), which were copolymerized with D,L-lactide to incorporate acryloyl groups in the copolymers. ${ }^{[27]}$ The acryloyl groups were amenable to the Michael-type addition reaction with varying thiol-containing molecules such as arginineglycine-aspartic acid-cysteine (RGDC) peptide under mild conditions. Kimura et al. synthesized a cyclic diester monomer consisting of glycolate and benzyl $\alpha$-L-malate units and copolymerized it with s-lactide to obtain biodegradable polymers with carboxyl groups. ${ }^{[28,29]}$ Langer's group synthesized poly[(L-lactic acid)-co-(L-lysine)] with functional lysine residue, which was further coupled with RGD peptide. ${ }^{[30,31]}$

Although the copolymerization of lactide/glycolide with other monomers is effective to generate functional groups in the random copolymers, this method often changes the physical properties (e.g., crystallinity and mechanical strength) of the initial homopolymers as well. To address this, a number of poly ( $\alpha$-hydroxyacid)-based block and graft copolymers have been designed and synthesized. ${ }^{[32-38]}$ Poly(ethylene glycol) (PEG) is the most widely used segment that has been introduced to the poly( $\alpha$-hydroxyacids). Diblock, triblock, and multiblock copolymers of PL(G)A/PEG have been synthesized conveniently by ring-opening polymerization of lactide/glycolide in the presence of PEG and selected catalysts. ${ }^{[39-44]}$ However, the functional groups (hydroxyl or carboxyl groups) in PEG-containing block copolymers can only be found at the end of each PEG segment, and their content in these block copolymers is very low, which limits subsequent chemical modification. A few non-PEG block and graft copolymers have been reported. ${ }^{[45-50]}$ Our group recently designed and synthesized a series of biodegradable amphiphilic poly[hydroxyalkyl (meth)acrylate]-graft-poly(L-lactic acid) (PHAA-gPLLA) copolymers. ${ }^{[51]}$ These copolymers contain pendant hydroxyl groups in the copolymer chains and have been successfully fabricated into 3D nano-fibrous scaffolds. Biomimetic scaffolds made from these copolymers can be further functionalized, are more hydrophilic, and have faster degradation rates than the PLLA homopolymer, which are advantageous for certain tissue engineering applications.

Poly( $\varepsilon$-caprolactone) (PCL) is another poly( $\alpha$-hydroxyacid) that has been used for tissue engineering applications. Similar strategies were utilized to incorporate functional groups into PCL chains. ${ }^{[52-56]}$ For instance, functionalized PCL copolymers were synthesized by copolymerization of $\varepsilon$-caprolactone with $\alpha$-chloro- $\varepsilon$-caprolactone, followed by atom transfer radical addition to incorporate pendant hydroxyl, carboxyl, and epoxide groups. ${ }^{[52]}$ The pendant hydroxyl groups in the PCL copolymers were also obtained by copolymerization of $\varepsilon$-caprolactone with another monomer, 5-ethyleneketal-e-caprolactone, followed by a deacetylization step to reduce the ketone groups into hydroxyl groups. ${ }^{[57]}$ The syntheses of these functional comonomers, as well as the de-protection process, however, are often complex and tedious.

Other synthetic biodegradable polymers, including poly(3-hydroxybutyrate) (PHB), ${ }^{[58]}$ polyurethanes (PU), ${ }^{[59,60]}$ polycarbonate (PC), ${ }^{[61]}$ poly(ortho ester) (POE) ${ }^{[62]}$ poly(propylene fumarate) (PPF), ${ }^{[63]}$ and polyphosphazenes (PP), ${ }^{[64]}$ have also been used as a scaffolding biomaterials. However, there are significantly fewer reports of functionalizing these biomaterials compared to the poly( $\alpha$-hydroxyacids). Some of the examples include the syntheses of functionalized PC. ${ }^{[65-67]}$ For instance, pendant amino groups were incorporated into PC chains by polymerization of the cyclic carbonate monomer, (2-oxo-[1,3]-dioxan-5-yl)carbamic acid benzyl ester, followed by removal of the protective benzyloxycarbonyl groups. ${ }^{[56]}$ Further functionalization of the pendant amino groups was demonstrated by grafting with RGD peptides. Because this reaction route involves multiple steps, the synthetic efficiency should be considered.

Synthetic polypeptides are emerging as a class of appealing functional biomaterials due to their unique physical, chemical, and biological properties. ${ }^{[68]}$ A good example is the peptide amphiphiles (PAs) designed by Stupp and coworkers. ${ }^{[69,70]}$ These PAs have five specific structural features: (i) a long alkyl tail that conveys hydrophobic character to the molecule and makes the molecule amphiphilic; (ii) four consecutive cysteine residues that form disulfide bonds to stabilize the structure; (iii) a linker region of three glycine residues to provide the hydrophilic head group the flexibility from the rigid crosslinked region; (iv) a phosphorylated serine residue that interacts strongly with calcium ions intended to enhance mineralization; and (v) an RGD peptide to aid in cell adhesion. ${ }^{[70]}$ The PAs have been shown to self-assemble into nano-fibrous networks when the strong electrostatic repulsion between molecules is neutralized either by changing $\mathrm{pH}$ or by adding divalent ions. Furthermore, the amphiphilic nature of the molecules allows the specific presentation of hydrophilic peptide signals on the surfaces of the assembled nano-structures. However, like many other hydrogel materials, the formation of mechanically stable 3D geometry from these PAs needs to be addressed.

Incorporation of proteinase-sensitive motifs into biomaterials is an exciting approach to prepare cell-responsive functional biomaterials. ${ }^{[7]}$ Hubbell and coworkers provided a good example of engineering synthetic PEG-based hydrogels as cell-ingrowth matrices for tissue regeneration. ${ }^{[71,72]}$ The hydrogel networks contain pendant oligo-

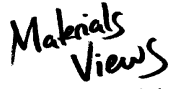

www.MaterialsViews.com 
peptides (RGDSP) for cell adhesion and matrix metalloproteinase (MMP) -sensitive peptides as crosslinkers for the PEG chains. The MMP-sensitive crosslinker determines the response of the material in the presence of cell-secreted MMPs. Therefore, this PEG-based hydrogel is a cellresponsive functional biomaterial. The authors further demonstrated that such gels are suitable matrices to deliver recombinant human bone morphogenetic protein-2 (rhBMP-2) and to induce bone regeneration.

\section{Surface Modification of 3D Polymer Scaffolds}

Generally, bioactive agents can be incorporated onto functionalized scaffolds through bulk or surface modification. In the bulk modification process, bioactive agents are coupled with functional polymers prior to scaffold fabrication. For example, the RGD peptide was first chemically attached to the lysine residue of poly[(L-lactic acid)-co-(Llysine)] before scaffold preparation. ${ }^{[31]}$ The RGD peptide, therefore, was distributed both on the surface and in the bulk of the poly[(L-lactic acid)-co-(L-lysine)] scaffold. Since cell/material interactions take place on scaffold surfaces, the bioactive molecules encapsulated inside the scaffold will not be able to interact with cells. Therefore, bulk modification is not an efficient way to incorporate bioactive agents. Furthermore, bulk modification often alters the mechanical and processing properties of the scaffold. In contrast, surface modification only presents bioactive agents on a scaffold surface; therefore, it can overcome the above limitations. In fact, this strategy has become increasingly attractive to prepare functional scaffolds. ${ }^{[73-77]}$

A number of surface modification methods have been developed to incorporate bioactive molecules onto the scaffold surface. A physical adsorption technique was reported to modify PLGA (75/25) scaffolds with either fibronectin or cell-adhesion motif RGD. ${ }^{[78]}$ While this surface coating process is simple, its efficiency is low and it has little control over the stability of the modified layer. Partial hydrolysis is a simple way to produce a certain amount of hydroxyl/carboxyl groups on the scaffold surface. ${ }^{[79-81]}$ For example, poly(D,L-lactic acid) (PDLLA) was treated under strong alkaline conditions to introduce hydroxyl groups, which were further used to graft chitosan. ${ }^{[82]}$ This method, however, is technique sensitive, and the hydrolysis also alters the surface morphology and bulk mechanical properties. Chemical vapor deposition (CVD) was originally developed to deposit thin films on solid substrates. ${ }^{[83-85]}$ This technique has recently been utilized to prepare surface-modification layers on the scaffold surface. ${ }^{[86-88]}$ For instance, poly [(4-amino- $p$-xylylene)-co-( $p$-xylylene)] was deposited on PCL surfaces through CVD polymerization to provide a reactive amine layer on the substrate surface. ${ }^{[87]}$ Biotin was then conjugated on the modified PCL surface to immobilize avidin for binding of biotinylated adenovirus. The biocompatibility of deposited poly[(4-amino- $p$-xylylene)-co-( $p$ xylylene)], however, has to be seriously considered. Plasma exposure is an effective procedure for surface etching. Oxygen plasma treatment was used to incorporate hydroxyl and peroxyl groups onto PLGA and PDLLA films. ${ }^{[89,90]}$ However, it has been reported that this plasma etching process alters both surface chemistry and surface morphology, which leads to the difficulty to predict how cells will respond on this surface-modified material. ${ }^{[89]}$ Plasma exposure has also been utilized to incorporate other functional groups on polymer backbones ${ }^{\left[{ }^{[1]}\right.}$ For instance, Nitschke et al. utilized low pressure ammonia plasma treatment for the modification of PHB thin films. ${ }^{[92]}$ The introduction of amine function was used for subsequent protein immobilization. The plasma treatment of PHB induced a durable conversion from a hydrophobic into a hydrophilic surface without significantly altering the morphology. Cheng and Teoh reported an argon plasma treatment for the modification of PCL thin films. ${ }^{\left[{ }^{[3]}\right.}$ The pretreated films were UV polymerized with acrylic acid prior to immobilization of collagen. Because of the limited plasma penetration, this method can only be used for twodimensional (2D) films or very thin 3D structures.

As discussed above, most of the surface modification techniques in the literature have been focusing on the modification of 2D films or very thin 3D constructs, and are limited for a 3D scaffold with designated structure and morphology. In our group, several effective techniques, including molecular entrapment and self-assembly, have been developed to modify the internal pore surfaces of 3D porous polymer scaffolds. ${ }^{[94-97]}$ For example, we developed a surface-entrapment technique to incorporate biomolecules (e.g., gelatin) onto the scaffold surface. ${ }^{[94]}$ The essential point of this technique was the selection of a suitable solvent system such that biomolecules were soluble in the solvent mixture, while the scaffold swelled but did not dissolve in the solvent mixture. As a non-solvent of the scaffold was added, the scaffold surface quickly shrank, leading to the entrapment of biomolecules on the surface of the scaffold. Compared with simple surface coating, the entrapped biomolecules were stable and did not dissolve when rinsed in water or aqueous tissue culture medium. No functional groups on the scaffold surfaces are needed for this surface modification method as long as proper solvent system is selected. In addition, the entrapment method can be used for any geometry, morphology, and thickness of 3D scaffold, which is a limitation for many other surface modification methods.

By integrating surface-entrapment into the scaffolding fabrication process, a porogen-induced surface modifica- 
tion technique has been developed to prepare biomimetic nano-fibrous PLLA scaffolds. ${ }^{[95]}$ In this one-step process, the modifying agent also acts as the porogen for the scaffold fabrication. Therefore, the surface modification is accomplished during the scaffold preparation. Gelatin was used as an example of a surface modification agent. First, gelatin spheres were fabricated and assembled into a 3D negative replica of the PLLA scaffold. The PLLA solution in water/ tetrahydrofuran (THF) mixture was then cast on the gelatin spheres template. The introduction of the solvent mixture in the PLLA solution was to ensure that certain amounts of gelatin molecules could be entrapped onto the surface of the PLLA scaffolds during the phase separation process. After phase separation and porogen removal, a nano-fibrous PLLA scaffold was generated with an interconnected spherical pore network, and its surface was modified with a layer of gelatin molecules.

Electronic layer-by-layer self-assembly has been widely used to prepare well-defined multilayer films. ${ }^{[98]}$ This process has recently been utilized to prepare functionalized scaffold surfaces in our group. ${ }^{[96]}$ In a typical process, the pretreated nano-fibrous PLLA scaffold was activated in an aqueous poly(diallyldimethylammonium chloride) (PDAC) solution to obtain a positively charged surface. After washing with water, the scaffold was subsequently immersed in a solution of negatively charged biomacromolecules (e.g., gelatin). Further growth of the PDAC/ gelatin bilayers was accomplished by repeating these procedures. To visualize the attachment of gelatin on the nano-fibrous scaffold surface, tetramethylrhodamine isothiocyanate (TRITC)-labeled gelatin was added in the selfassembly solution. Development of color on the scaffold after the self-assembly process showed that the gelatin was successfully incorporated on the nano-fibrous PLLA scaffold surface (Figure 1a). Confocal images further confirmed that gelatin was distributed evenly throughout the entire scaffold surface (Figure $1 \mathrm{~b}$ and $\mathrm{c}$ ). This self-assembly technique has a high degree of molecular control over surface chemistry, coating thickness, and maintenance of the 3D scaffold architecture. Moreover, the use of aqueous solutions makes it easy to carry out. Therefore, it is an appealing surface modification method for 3D scaffolds with complex geometry as long as the scaffold pores are interconnected.

In order to mimic both the physical architecture and chemical composition of natural extracellular bone matrix, a biomimetic process that allows in situ apatite formation on the internal surfaces of the pore walls of nano-fibrous gelatin scaffolds was developed using a simulated body fluid (SBF) technique. ${ }^{[97]}$ A large number of nano-featured bone-like apatite microparticles were grown on the internal surfaces of nano-fibrous gelatin scaffolds after $7 \mathrm{~d}$ of incubation (Figure 2c and d). Control over the particle size and their coverage of the pore surfaces was achieved by a)

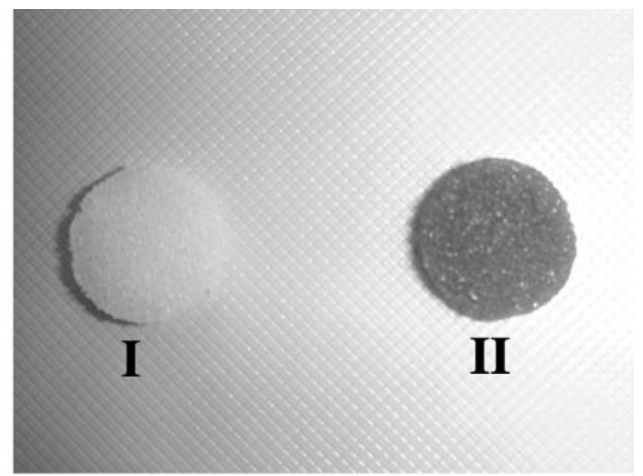

b)

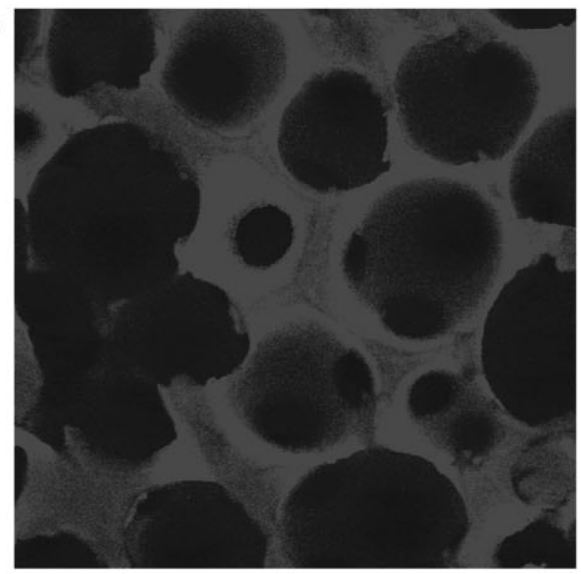

c)

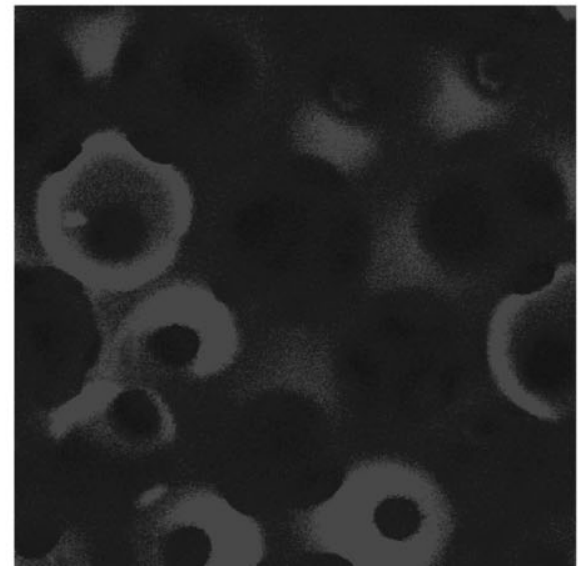

Figure 1. Surface modification of a 3D nano-fibrous PLLA with gelatin. TRITC-labeled gelatin was utilized to visualize the attachment and distribution of gelatin on the scaffold surface. (a) A photograph shows the overview of the scaffold before (I) and after surface modification (II); (b) a confocal image shows the distribution of gelatin on the outermost surface after surface modification; (c) a confocal image shows the distribution of gelatin in the center of the scaffold after surface modification. From Liu et al., ${ }^{[107]}{ }^{(} 2009$ by Springer.

adjusting the incubation conditions such as SBF concentration, incubation time, and $\mathrm{pH}$ value of the solution. Generally, a longer incubation time of the NF-gelatin scaffold led to more apatite formation (Figure 2e). However,

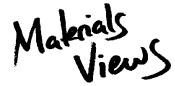

www.MaterialsViews.com 
a)

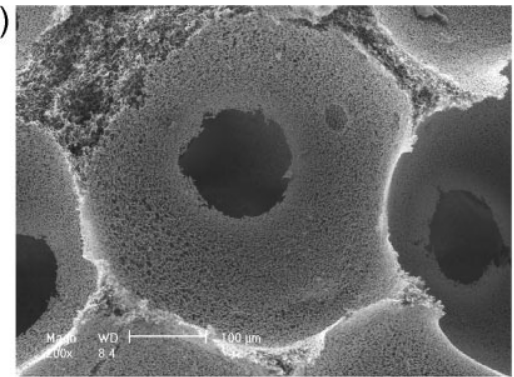

b)

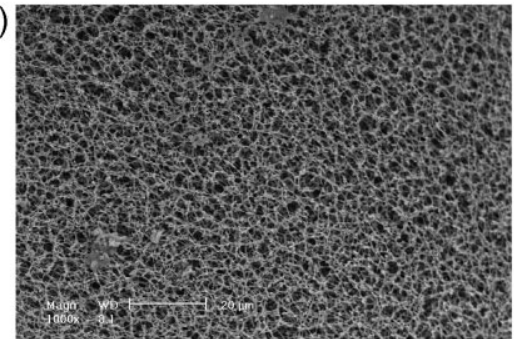

c)

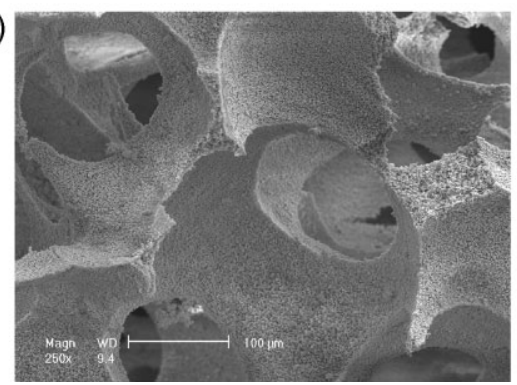

d)

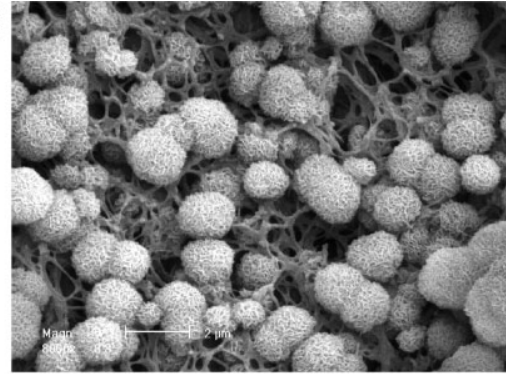

e)

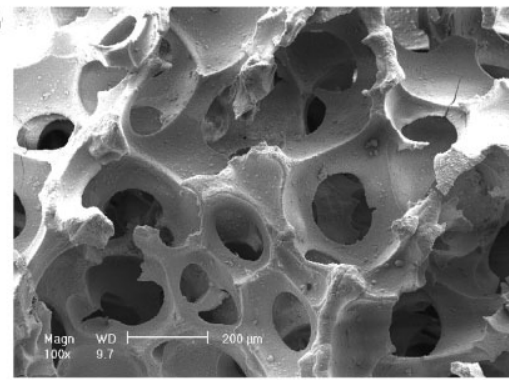

f)

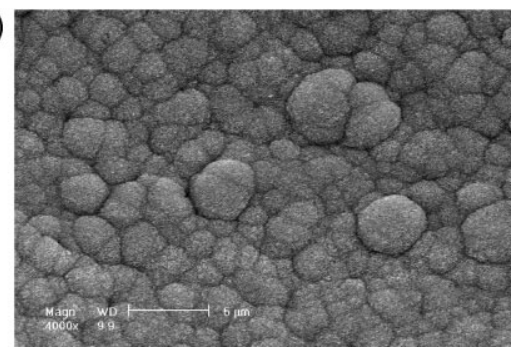

Figure 2. SEM images of nano-fibrous gelatin scaffolds with surface modification of apatite particles. The apatite microparticles were prepared by incubated in $1.5 \times \mathrm{SBF}$ for varying times. (a) $1 \mathrm{~d}$ overview; (b) $1 \mathrm{~d}$ pore/wall structure; (c) $7 \mathrm{~d}$ overview; (d) $7 \mathrm{~d}$ pore/ wall structure; (e) $21 \mathrm{~d}$ overview; (f) $21 \mathrm{~d}$ pore/wall structure. From Liu et al.,, ${ }^{[97]} \bigcirc 2009$ by Elsevier.

the interconnected macroporous structure of the scaffolds was still maintained, which is important for cell migration and mass transport when used for tissue regeneration. In addition to the aimed surface chemical modification for improved osteoconductivity, the mechanical properties of the surface-modified nano-fibrous gelatin scaffolds were also significantly improved compared to the plain gelatin scaffolds.

\section{Cell Response on Functionalized Scaffolds}

Functionalizing the scaffold with carboxylic acid groups has shown promise by making the surface more hydrophilic. Fibroblasts seeded on carboxylic acid modified PGA, PLGA, and PLLA scaffolds were spread over a larger area and had higher adhesion and proliferation rates compared to unmodified scaffolds. ${ }^{[99]}$ Incorporation of hydroxyapatite (HA) into scaffolds has been shown to significantly increase

osteoblast adhesion and proliferation compared to pure PLLA scaffolds. ${ }^{[100]}$

As discussed above, a more reliable method to functionalize scaffolds is to use bioactive molecules derived from natural ECM proteins. For instance, gelatin, a denatured form of collagen, was used to modify the surfaces of 3D PLLA scaffolds. ${ }^{[95,96]}$ The presence of gelatin greatly increased the adherence, proliferation, and spreading of MC3T3-E1 cells and more collagen fibers and other cell secretions were deposited on the surfacemodified scaffolds than on the control scaffolds (Figure 3) $\cdot{ }^{[94]}$ More specific than gelatin, peptide fragments have been used to tailor cell adhesion and growth. The RGD peptide is ubiquitous in the ECM and promotes cell adhesion by acting as a binding site for integrins. ${ }^{[01,102]}$ PCL films modified with RGD served to promote the attachment and spreading of mesenchymal stem cells (MSCs). ${ }^{[101]}$ RGD may increase focal adhesion kinase (FAK) phosphorylation for integrin-based signal transduction, which could play a role in cell proliferation and viability. ${ }^{[03]}$

In addition to adhesion and proliferation, functionalized scaffolds can be used to direct cell differentiation. The incorporation of HA on gelatin scaffolds was used to study the differentiation of MC3T3-E1 preosteoblasts into osteoblasts. ${ }^{\left[{ }^{[7]}\right.}$ Cells were seeded on gelatin scaffolds with and without HA for 4 weeks and two osteogenic markers [bone sialoprotein (BSP) and osteocalcin $(\mathrm{OCN})]$ were examined. Results showed that levels of BSP and OCN were five and two times higher, respectively, in the gelatin-HA scaffolds compared to the gelatin scaffolds. The use of HA thus not only enhances the mechanical strength of the scaffold, but it also plays a role in cell differentiation and tissue formation.

The most widely used method to induce cell differentiation is the delivery of growth factors from scaffolds. A variety of methods have been developed to incorporate growth factors into scaffolds. ${ }^{[11,104]}$ For example, recombinant human bone morphogenic protein 7 (rhBMP-7) was encapsulated in PLGA microspheres using a double emulsion technique, and the microspheres were incorporated onto the surface of a nano-fibrous PLLA scaffold. ${ }^{[105]}$ The biomimetic PLLA scaffolds incorporated with rhBMP-7 microspheres were found to significantly enhance bone formation. After 6 weeks of subcutaneous implantation in Sprague-Dawley rats, the scaffolds with rhBMP-7 micro- 
a)

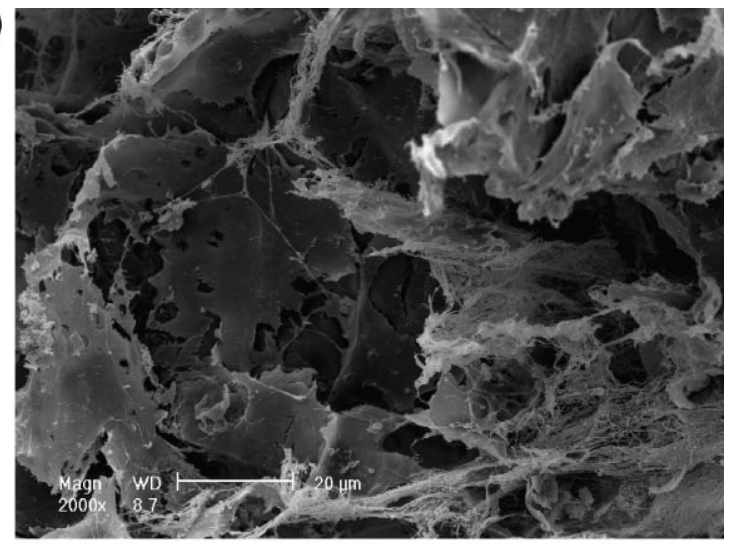

b)

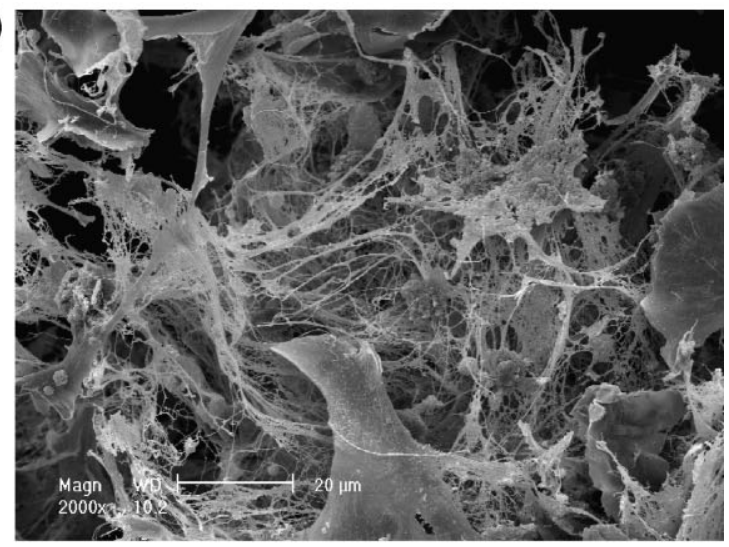

c)

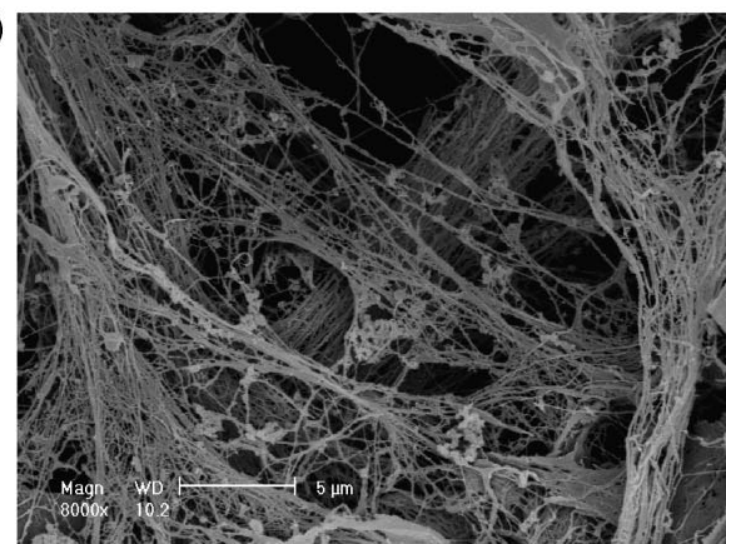

Figure 3. SEM images of PLLA scaffolds 4 weeks after cell seeding: (a) PLLA controls; (b) surface-modified PLLA scaffolds. The scaffold was physically entrapped with gelatin, followed by chemical crosslinking; (c) high magnification of (b). Significantly higher amount of collagen fibers and other cell secretions were deposited on the surface-modified scaffolds than on the control scaffolds. From Liu et al., ${ }^{[94]}{ }^{\circ} 2005$ by Wiley Periodicals, Inc.

spheres showed significantly more bone formation than scaffolds with rhBMP-7 simply adsorbed on the surface. This study illustrates the important role that growth factors can play in a tissue engineering strategy.

While the most common method is to use growth factors to induce differentiation and tissue neogenesis, small molecules are also capable of guiding cell fate through cell/matrix interactions. Recent work has shown that the fate of human MSCs could be changed when they were seeded on hydrogels with various functional groups. ${ }^{[106]}$ Carboxylic acid groups, mimicking cartilage glycosaminoglycans, increased the expression of aggrecan, an indicator of chondrogenic differentiation. Phosphate groups, important for mineralized tissue formation, increased expression levels of CBFA1, a marker for osteogenic differentiation. Finally, $t$-butyl groups, mimicking lipid filled adipocytes, increased expression of peroxisome proliferating antigen receptor gamma (PPARG), a measure of adipogenic differentiation. The ability of small molecules to induce differentiation shows potential as an easier, cheaper, and safer way to guide the fate of stem cells, yet more work needs to be done to validate their efficacy when compared to the use of growth factors.

\section{Conclusion}

The development of tissue engineering has entered a new phase in which rational design is being used to produce functionalized biomaterials and scaffolds tailored to specific applications. These functionalized scaffolds are no longer just a physical environment with little or no effect on the regeneration process. Rather, they are active participants in the tissue neogenesis process.

Future research will continue to increase the active role of the scaffold in the regeneration process and to elucidate the mechanisms in which functionalized scaffolds influence cell differentiation, proliferation, and morphology. The polymer synthesis, scaffold fabrication, surface functionalization, and growth factor delivery could be combined to create more advanced scaffolds tailored to specific applications.

Received: November 17, 2011; Published online: March 6, 2012; DOI: $10.1002 / \mathrm{mabi} .201100466$

Keywords: biodegradable polymers; cell adhesion; scaffolds; surface modification; tissue engineering

[1] R. Langer, J. P. Vacanti, Science 1993, 260, 920

[2] P. X. Ma, Adv. Drug Delivery Rev. 2008, 60, 184.

[3] X. H. Liu, P. X. Ma, Ann. Biomed. Eng. 2004, 32, 477.

[4] R. Langer, D. A. Tirrell, Nature 2004, 428, 487.

[5] J. Kohn, Nat. Mater. 2004, 3, 745.

[6] J. A. Hubbell, Curr. Opin. Biotechnol. 1999, 10, 123.

[7] M. P. Lutolf, J. A. Hubbell, Nat. Biotechnol. 2005, 23, 47.

[8] L. Moroni, P. Habibovic, D. J. Mooney, C. A. van Blitterswijk, MRS Bull. 2010, 35, 584.

[9] M. Niinomi, J. Artif. Org. 2008, 11, 105

[10] C. Ohtsuko, M. Kamitakahara, T. Miyazaki, J. R. Soc. Interface 2009, 6, S349.

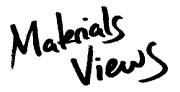

www.MaterialsViews.com 
[11] G. Wei, P. X. Ma, Adv. Funct. Mater. 2008, 18, 3568.

[12] K. Rezwan, O. Z. Chen, J. J. Blaker, A. R. Boccaccini, Biomaterials 2006, 27, 3413.

[13] S. F. Yang, K. F. Leong, Z. H. Du, C. K. Chua, Tissue Eng. 2002, $8,1$.

[14] S. F. Yang, K. F. Leong, Z. H. Du, C. K. Chua, Tissue Eng. 2001, 7, 679.

[15] L. A. Smith, X. H. Liu, P. X. Ma, Soft Matter 2008, 4, 2144.

[16] C. M. Agrawal, R. B. Ray, J. Biomed. Mater. Res. 2001, 55, 141.

[17] J. K. Tessmar, A. M. Gopferich, Adv. Drug Delivery Rev. 2007, 59, 274.

[18] M. J. Whitaker, R. A. Quirk, S. M. Howdle, K. M. Shakesheff, J. Pharm. Pharmacol. 2001, 53, 1427.

[19] P. X. Ma, J. W. Choi, Tissue Eng. 2001, 7, 23.

[20] P. X. Ma, R. Y. Zhang, J. Biomed. Mater. Res. 1999, 46, 60.

[21] K. M. Woo, V. J. Chen, P. X. Ma, J. Biomed. Mater. Res., Part A 2003, 67A, 531.

[22] K. M. Woo, J. Seo, R. Y. Zhang, P. X. Ma, Biomaterials 2007, 28 , 2622.

[23] L. S. Nair, C. T. Laurencin, Prog. Polym. Sci. 2007, 32, 762.

[24] B. He, J. Z. Bei, S. G. Wang, Polymer 2003, 44, 989.

[25] M. Leemhuis, C. F. van Nostrum, J. A. W. Kruijtzer, Z. Y. Zhong, M. R. ten Breteler, P. J. Dijkstra, J. Feijen, W. E. Hennink, Macromolecules 2006, 39, 3500.

[26] D. E. Noga, T. A. Petrie, A. Kumar, M. Weck, A. J. Garcia, D. M. Collard, Biomacromolecules 2008, 9, 2056.

[27] W. Chen, H. C. Yang, R. Wang, R. Cheng, F. H. Meng, W. X. Wei, Z. Y. Zhong, Macromolecules 2010, 43, 201.

[28] Y. Kimura, K. Shirotani, H. Yamane, T. Kitao, Polymer 1993, 34, 1741.

[29] Y. Kimura, K. Shirotani, H. Yamane, T. Kitao, Macromolecules 1988, 21, 3338.

[30] D. A. Barrera, E. Zylstra, P. T. Lansbury, R. Langer, J. Am. Chem. Soc. 1993, 115, 11010.

[31] A. D. Cook, J. S. Hrkach, N. N. Gao, I. M. Johnson, U. B. Pajvani, S. M. Cannizzaro, R. Langer, J. Biomed. Mater. Res. 1997, 35, 513.

[32] C. Deng, H. Y. Tian, P. B. Zhang, J. Sun, X. S. Chen, X. B. Jing, Biomacromolecules 2006, 7, 590.

[33] B. Jeong, Y. H. Bae, S. W. Kim, Macromolecules 1999, 32, 7064.

[34] F. Unger, M. Wittmar, T. Kissel, Biomaterials 2007, 28, 1610.

[35] U. Westedt, M. Wittmar, M. Hellwig, P. Hanefeld, A. Greiner, A. K. Schaper, T. Kissel, J. Controlled Release 2006, 111, 235.

[36] Y. Q. Wang, D. E. Noga, K. Yoon, A. M. Wojtowicz, A. S. P. Lin, A. J. Garcia, D. M. Collard, M. Weck, Adv. Funct. Mater. 2008, 18, 3638.

[37] F. F. Wolf, N. Friedemann, H. Frey, Macromolecules 2009, 42, 5622.

[38] F. K. Wolf, H. Frey, Macromolecules 2009, 42, 9443.

[39] X. H. Chen, S. P. McCarthy, R. A. Gross, Macromolecules 1997, 30, 4295.

[40] C. Deng, G. Z. Rong, H. Y. Tian, Z. H. Tang, X. S. Chen, X. B. Jing, Polymer 2005, 46, 653.

[41] T. Fujiwara, T. Mukose, T. Yamaoka, H. Yamane, S. Sakurai, Y. Kimura, Macromol. Biosci. 2001, 1, 204.

[42] S. M. Li, M. Vert, Macromolecules 2003, 36, 8008.

[43] I. Rashkov, N. Manolova, S. M. Li, J. L. Espartero, M. Vert, Macromolecules 1996, 29, 50.

[44] Y. O. Wan, W. N. Chen, J. Yang, J. Z. Bei, S. G. Wang, Biomaterials 2003, 24, 2195.

[45] Y. N. Yang, J. Cai, X. L. Zhuang, Z. P. Guo, X. B. Jing, X. S. Chen, Polymer 2010, 51, 2676.

[46] Y. X. Li, J. Nothnagel, T. Kissel, Polymer 1997, 38, 6197.
[47] C. Nouvel, P. Dubois, E. Dellacherie, J. L. Six, J. Polym. Sci., Part A: Polym. Chem. 2004, 42, 2577.

[48] F. S. Palumbo, G. Pitarresi, D. Mandracchia, G. Tripodo, G. Giammona, Carbohydr. Polym. 2006, 66, 379.

[49] Y. Teramoto, Y. Nishio, Polymer 2003, 44, 2701.

[50] Y. Li, O. B. Li, F. X. Li, H. Y. Zhang, L. Jia, J. Y. Yu, O. Fang, A. Cao, Biomacromolecules 2006, 7, 224.

[51] X. H. Liu, P. X. Ma, Biomaterials 2010, 31, 259.

[52] R. Riva, S. Lenoir, R. Jerome, P. Lecomte, Polymer 2005, 46, 8511.

[53] J. Rieger, K. Van Butsele, P. Lecomte, C. Detrembleur, R. Jerome, C. Jerome, Chem. Commun. 2005, 274.

[54] C. Detrembleur, M. Mazza, O. Halleux, P. Lecomte, D. Mecerreyes, J. L. Hedrick, R. Jerome, Macromolecules 2000, 33, 14.

[55] S. Gautier, V. D’Aloia, O. Halleux, M. Mazza, P. Lecomte, R. Jerome, J. Biomater. Sci. -Polym. Ed. 2003, 14, 63.

[56] X. L. Hu, X. S. Chen, Z. G. Xie, H. B. Cheng, X. B. Jing, J. Polym. Sci., Part A: Polym. Chem. 2008, 46, 7022.

[57] D. Tian, P. Dubois, C. Grandfils, R. Jerome, Macromolecules 1997, 30, 406

[58] G. O. Chen, O. Wu, Biomaterials 2005, 26, 6565.

[59] J. P. Santerre, K. Woodhouse, G. Laroche, R. S. Labow, Biomaterials 2005, 26, 7457.

[60] J. J. Guan, K. L. Fujimoto, M. S. Sacks, W. R. Wagner, Biomaterials 2005, 26, 3961.

[61] S. J. Lee, J. S. Choi, K. S. Park, G. Khang, Y. M. Lee, H. B. Lee, Biomaterials 2004, 25, 4699.

[62] J. Heller, J. Barr, S. Y. Ng, K. S. Abdellauoi, R. Gurny, Adv. Drug Delivery Rev. 2002, 54, 1015.

[63] J. P. Fisher, J. W. M. Vehof, D. Dean, J. van der Waerden, T. A. Holland, A. G. Mikos, J. A. Jansen, J. Biomed. Mater. Res. 2002, 59, 547.

[64] M. T. Conconi, S. Lora, A. M. Menti, P. Carampin, P. P. Parnigotto, Tissue Eng. 2006, 12, 811.

[65] X. L. Hu, S. Liu, X. S. Chen, G. J. Mo, Z. G. Xie, X. B. Jing, Biomacromolecules 2008, 9, 553.

[66] R. Wu, T. F. Al-Azemi, K. S. Bisht, Biomacromolecules 2008, 9 , 2921.

[67] Y. Zhou, R. X. Zhuo, Z. L. Liu, Macromol. Rapid Commun. 2005, $26,1309$.

[68] S. G. Zhang, Nat. Biotechnol. 2003, 21, 1171.

[69] J. D. Hartgerink, E. Beniash, S. I. Stupp, Science 2001, 294, 1684.

[70] J. D. Hartgerink, E. Beniash, S. I. Stupp, Proc. Natl. Acad. Sci. USA 2002, 99, 5133.

[71] M. P. Lutolf, J. L. Lauer-Fields, H. G. Schmoekel, A. T. Metters, F. E. Weber, G. B. Fields, J. A. Hubbell, Proc. Natl. Acad. Sci. USA 2003, 100, 5413.

[72] M. P. Lutolf, G. P. Raeber, A. H. Zisch, N. Tirelli, J. A. Hubbell, Adv. Mater. 2003, 15, 888

[73] Z. W. Ma, C. Y. Gao, Y. H. Gong, J. C. Shen, Biomaterials 2005, 26,1253

[74] Z. W. Ma, W. He, T. Yong, S. Ramakrishna, Tissue Eng. 2005, 11, 1149.

[75] L. Y. Santiago, R. W. Nowak, J. P. Rubin, K. G. Marra, Biomaterials 2006, 27, 2962.

[76] J. Yang, G. X. Shi, J. Z. Bei, S. G. Wang, Y. L. Cao, O. X. Shang, G. G. Yang, W. J. Wang, J. Biomed. Mater. Res. 2002, 62, 438.

[77] J. J. Yoon, S. H. Song, D. S. Lee, T. G. Park, Biomaterials 2004, 25, 5613.

[78] X. B. Yang, H. I. Roach, N. M. P. Clarke, S. M. Howdle, R. Quirk, K. M. Shakesheff, R. O. C. Oreffo, Bone 2001, 29, 523. 
[79] J. M. Gao, L. Niklason, R. Langer, J. Biomed. Mater. Res. 1998, $42,417$.

[80] K. Y. Cai, K. D. Yao, X. Hou, Y. Q. Wang, Y. J. Hou, Z. M. Yang, X. O. Li, H. O. Xie, J. Biomed. Mater. Res. 2002, 62, 283.

[81] T. I. Croll, A. J. O'Connor, G. W. Stevens, J. J. Cooper-White, Biomacromolecules 2004, 5, 463.

[82] K. Y. Cai, K. D. Yao, Y. L. Cui, S. B. Lin, Z. M. Yang, X. O. Li, H. O. Xie, T. W. Qing, J. Luo, J. Biomed. Mater. Res. 2002, 60, 398.

[83] M. E. Alf, A. Asatekin, M. C. Barr, S. H. Baxamusa, H. Chelawat, G. Ozaydin-Ince, C. D. Petruczok, R. Sreenivasan, W. E. Tenhaeff, N. J. Trujillo, S. Vaddiraju, J. J. Xu, K. K. Gleason, Adv. Mater. 2010, 22, 1993.

[84] M. L. Ma, Y. Mao, M. Gupta, K. K. Gleason, G. C. Rutledge, Macromolecules 2005, 38, 9742.

[85] M. Leskela, H. Molsa, L. Niinisto, Supercond. Sci. Technol. 1993, 6, 627

[86] J. Lahann, M. Balcells, T. Rodon, J. Lee, I. S. Choi, K. F. Jensen, R. Langer, Langmuir 2002, 18, 3632.

[87] W. W. Hu, Y. Elkasabi, H. Y. Chen, Y. Zhang, J. Lahann, S. J. Hollister, P. H. Krebsbach, Biomaterials 2009, 30, 5785.

[88] Y. Zhang, X. P. Deng, E. L. Scheller, T. G. Kwon, J. Lahann, R. T. Franceschi, P. H. Krebsbach, Biomaterials 2010, 31 , 3231.

[89] Y. O. Wang, X. Qu, J. Lu, C. F. Zhu, L. J. Wan, J. L. Yang, J. Z. Bei, S. G. Wang, Biomaterials 2004, 25, 4777.

[90] J. Yang, J. Z. Bei, S. G. Wang, Biomaterials 2002, 23, 2607.

[91] P. K. Chu, J. Y. Chen, L. P. Wang, N. Huang, Mater. Sci. Eng. R-Rep. 2002, 36, 143.
[92] M. Nitschke, G. Schmack, A. Janke, F. Simon, D. Pleul, C. Werner, J. Biomed. Mater. Res. 2002, 59, 632.

[93] Z. Y. Cheng, S. H. Teoh, Biomaterials 2004, 25, 1991.

[94] X. H. Liu, Y. J. Won, P. X. Ma, J. Biomed. Mater. Res., Part A 2005, 74A, 84.

[95] X. H. Liu, Y. J. Won, P. X. Ma, Biomaterials 2006, 27, 3980.

[96] X. H. Liu, L. Smith, G. B. Wei, Y. J. Won, P. X. Ma, J. Biomed. Nanotechnol. 2005, 1, 54

[97] X. Liu, L. A. Smith, J. Hu, P. X. Ma, Biomaterials 2009, 30, 2252.

[98] G. Decher, Science 1997, 277, 1232.

[99] K. Park, Y. M. Ju, J. S. Son, K.-D. Ahn, D. K. Han, J. Biomater. Sci. -Polym. Ed. 2007, 18, 369.

[100] P. X. Ma, R. Y. Zhang, G. Z. Xiao, R. Franceschi, J. Biomed. Mater. Res. 2001, 54, 284

[101] U. Hersel, C. Dahmen, H. Kessler, Biomaterials 2003, 24, 4385.

[102] M. D. Pierschbacher, E. Ruoslahti, Nature 1984, 309, 30

[103] H. Zhang, S. Hollister, J. Biomater. Sci. 2009, 20, 1975.

[104] J. E. Babensee, L. V. McIntire, A. G. Mikos, Pharm. Res. 2000, 17, 497.

[105] G. Wei, O. Jin, W. V. Giannobile, P. X. Ma, Biomaterials 2007, 28, 2087.

[106] D. S. W. Benoit, M. P. Schwartz, A. R. Durney, K. S. Anseth, Nat. Mater. 2008, 7, 816.

[107] X. Liu, I. O. Smith, P. X. Ma, "Biomimetic Nanophase Materials to Promote New Tissue Formation for Tissueengineering Applications", in Biological Interactions on Materials Surfaces, Eds., D. A. Puleo, R. Bizios), Springer, New York 2009, pp. 283-296. 